\title{
CUATRO ESPECIES Y UNA VARIEDAD DEL GENERO ASTROSPHAERIELLA (DOTHIDEALES, MELANOMMATACEAE) DE MEXICO
}

\author{
Felipe Eduardo San Martin
}

Y

Pablo Antonio LaVin

Biodiversidad Tamaulipeca, A.C.

Sierra Hermosa 617

Fracc. Villa Real

87010 Cd. Victoria, Tamaulipas

\section{RESUMEN}

Se describen e ilustran Astrosphaeriella stellata (Pat.) Sacc., A. stellata var. palmicola var. nov., A. tornata (Berk. et M. A. Curtis) D. Hawksw. et Boise, A. trochus (Penzig et Sacc.) D. Hawksw. y $A$. vaginata sp. nov. Se provee una clave artificial para los taxa considerados.

\section{ABSTRACT}

Astrosphaeriella stellata (Pat.) Sacc., A. stellata var. palmicola var. nov., A. tornata (Berk. et M. A. Curtis) D. Hawksw. et Boise, A. trochus (Penzig et Sacc.) D. Hawksw., and A. vaginata sp. nov., are described and illustrated. An artificial key is provided for the considered taxa.

\section{INTRODUCCION}

Astrosphaeriella Sydow et P. Sydow pertenece al orden Dothideales sensu Barr, familia Melanommataceae G. Winter de la antigua serie Loculoascomycetes (Hawksworth et al., 1995). Para Courtecuisse et al. (1996) el género debe asignarse a la familia Platystomaceae de los Melannomatales. En cualquier caso, Astrosphaeriella se caracteriza por sus ascomas comúnmente solitarios o rara vez 2 ó 3 unidos por sus bases; al principio inmersos y subepidérmicos, después superficiales por irrupción a partir, por lo general, de madera dura de monocotiledóneas como palmas y bambusáceas. En la madurez los ascomas pueden permanecer cubiertos por la epidermis del hospedante con excepción de la región ostiolar o el tejido romperse quedando como bandas o lengüetas más o menos triangulares alrededor de la base, lo que le da al conjunto una apariencia estrellada. Los ascomas individuales son uniloculares, no estromáticos, peritecioides, hemisféricos a cónicos, con la base plana, ostiolados, de color café, café obscuro o casi negro; peridio de apariencia carbonosa, grueso, compuesto de células de color café-rojizo, pseudoparenquimáticas, de textura angular, sensu Starback (1895), y con el lumen hialino; 
la base de la cavidad ascomática por lo regular se desarrolla pobremente, de tal forma que parece que ascos y pseudoparáfisis están en contacto con el substrato. Ostiolo negruzco, mamiforme, sin perífisis. Pseudoparáfisis trabeculadas, filiformes, persistentes, septadas, ramificadas y anastomosadas, embebidas junto con ascos y ascosporas en una matriz sol-gel dextrinoide. Los ascos nacen a partir del pseudotejido ascomático basal que puede ser escaso o bien desarrollado, cilíndricos, con pedicelos por lo regular cortos, bitunicados, con el ápice de la túnica interna constreñida, octosporados. Las ascosporas se presentan arregladas en el asco en forma parcialmente biseriada a biseriada, alargadas, fusiformes, rectas o algo arqueadas, adelgazándose gradualmente hacia los extremos, con 1-5 septos, a veces con constricciones en la región septal, hialinas o de color café-pálido o café-rojizo, lisas o estriadas, con los ápices más claros, gutuladas, cubiertas por una envoltura hialina más evidente en los propágulos jóvenes, sin poros o líneas germinales (Hawksworth, 1981; Aptroot, 1985; Hawksworth y Boise, 1985).

El género más cercano a Astrosphaeriella es Trematosphaeria Fuckel. El primero se separa del segundo porque coloniza monocotiledóneas, no dicotiledóneas y por sus ascosporas de color más pálido (Hawksworth, 1981; Boise, 1985). A juzgar por el hábito y la morfología de los ascomas, es probable que Astrosphaeriella y Trematosphaeria sean congenéricos, pero mientras no se cuente con datos anamórficos de ambos, no es posible concluir al respecto.

A la fecha, las 13 especies reconocidas de Astrosphaeriella han sido recolectadas en áreas tropicales de Africa, Asia, y Centro y Sudamérica.

En esta contribución se registran por vez primera para México Astrosphaeriella stellata (Pat.) Sacc., A. tornata (Berk. et M. A. Curtis) D. Hawks. y A. trochus (Penz. et Sacc.) D. Hawksw. Asimismo, se propone a Astrosphaeriella vaginata sp. nov. y A. stellata var. palmicola var. nov.

\section{METODOLOGIA}

La recolección del material fúngico, así como su herborización e identificación, se hizo de acuerdo con San Martín (1996). Los acrónimos de los herbarios (con excepción de FSG cuyo registro está en trámite) fueron tomados de Holmgren et al. (1990).

\section{CLAVE PARA LAS ESPECIES DE ASTROSPHAERIELLA CONSIDERADAS EN ESTE ESTUDIO}

1 Ascomas con restos del substrato aheridos a sus paredes. Ascosporas oliváceas a amarillentas, de (46-)49.5-56(-57) x 6.5-7(-9) $\mu \mathrm{m}$, uniseptadas, con una envoltura hialina persistente en la madurez. En madera de Bactris sp.

Astrosphaeriella vaginata sp. nov.

1 Ascomas con restos del substrato en la base a manera de bandas o lengüetas triangulares reflejas que dan la apariencia de una estrella. Combinación de caracteres diferente 
2 Ascosporas pentaseptadas, de color oliváceo a café claro, de (59-)61.5-68 x (6-)6.5-7 $\mu \mathrm{m}$, fusiformes, alargadas a arqueadas, con una envoltura hialina persistente en la madurez. En madera de Chusquea sp. Astrosphaeriella trochus

2 Ascosporas uni o triseptadas. Combinación de caracteres diferente ........................ 3

3 Ascosporas triseptadas, de color café a café obscuro, de (50.5-)51-64.5(-65) x (8-)9-11 $\mu \mathrm{m}$, fusiformes, equilaterales o inequilaterales y algo arqueadas, constreñidas a la altura de los septos. En madera de una bambusoidea no identificada

Astrosphaeriella tornata

3 Ascosporas uniseptadas. Combinación de caracteres diferente 4

4 Ascosporas hialinas a amarillentas, de (37-)38.5-48 x (4-)5-5.5 $\mu \mathrm{m}$, fusiformes, equilaterales o inequilaterales, constreñidas a la altura del septo. En madera de Chusquea sp.

Astrosphaeriella stellata var. stellata

4 Ascosporas de color café, translúcidas, de (34-)35.5-43(-44) x 7-9 $\mu \mathrm{m}$, fusiformes, equilaterales o inequilaterales, constreñidas a la altura del septo. En madera de una palma no identificada Astrosphaeriella stellata var. palmicola var. nov.

\section{DESCRIPCIONES}

Astrosphaeriella stellata (Pat.) Sacc. var. stellata, Sylloge Fungorum 24: 938. 1928. Fig. 1A, B.

Ascomas individuales rara vez unidos por sus bases, hemisféricos a cónicos, de 0.4-0.5 mm de alto x 0.6-0.8 mm de diámetro, errumpentes, con restos de epidermis del hospedante en la base a manera de bandas o lengüetas reflejas, dándole al conjunto la apariencia de una estrella. Textura carbonosa. Superficie lisa. Externamente de color café obscuro a negruzo con el área papilar más obscura, internamente negruzcos. Pseudohimenio colapsado, de 0.4-0.5 mm de diámetro. Ostiolos papilados. Ascos bitunicados, con la túnica interna constreñida en su parte superior, cilíndricos, octosporados en forma parcialmente biseriada, con pedicelos cortos, de 121-140 $\mu \mathrm{m}$ de longitud total $\mathrm{x}$ 11.5-13 $\mu \mathrm{m}$ de ancho, la parte esporígena de 112-131 $\mu \mathrm{m}$, sin anillo apical o éste muy reducido y en tal caso inamiloide. Pseudoparáfisis trabeculadas, septadas, numerosas, de ca. $1 \mu \mathrm{m}$ de ancho, incluidas en una matriz gelatinosa. Ascosporas maduras hialinas a amarillentas, de (37-)38.5-48 x (4-)5-5.5 $\mu \mathrm{m}$, fusiformes, equilaterales o inequilaterales con extremos aguzados, uniseptadas, constreñidas a la altura del septo.

Especímenes estudiados: Chiapas, ejido El Rosario, municipio de Motozintla, 2000 m s.n.m., en madera de Chusquea sp. en un bosque tropical con Pinus-Quercus, 19.X.1997, San Martín 6714Ch (SMG). 1981).

Observaciones: el material arriba descrito es típico de la especie (véase Hawksworth,

Astrosphaeriella stellata ha sido recolectada en la India, Japón, Java, las Filipinas, Vietnam (Hawksworth, 1981) y en la Guyana Francesa (Cortecuisse et al., 1996). En Japón causa una enfermedad en bambú caracterizada por la decoloración de la madera (Hawksworth, 1981). 
Astrosphaeriella stellata (Pat.) Sacc. var. palmicola var. nov. Fig. 1C, D, E.

A varietate typica speciei differt hospite quod palma est, ascomatibus majoribus i. e. 0.7-0.9 mm diametro $\times$ 0.8-1 mm altis vs. 0.6-0.8 $\mathrm{mm}$ diametro $\times$ 0.4-0.5 altis, ascosporis brunneis potius hyalinis vel flavidis, exigue brevioribus et latioribus i. e. (34-)35.5-43(-44) x (6.5-)7-9 $\mu \mathrm{m}$ vs. (37-)38.5-48 x (4-)5-5.5 $\mu \mathrm{m}$.

Especímenes estudiados: Chiapas, ejido Boca de Chajul, municipio de Ocosingo, en madera de la palma "chocho" o "coyolillo" en un acahual, 26.V.1988, San Martín 871 (HOLOTIPO: ITCV); ibid., reserva de la biosfera Montes Azules, municipio de Ocosingo, en madera de la palma "chocho" o "coyolillo" en una selva alta perennifolia, 28.V.1988, San Martín 814Ch y 816 (ITCV).

Observaciones: este taxon difiere de la variedad típica en que su hospedante es una palma y no una gramínea bambusoide, los ascomas son más grandes i. e. de 0.7-0.9 $\mathrm{mm}$ de ancho $\times 0.8-1 \mathrm{~mm}$ de alto vs. $0.6-0.8 \mathrm{~mm}$ de ancho $\times 0.4-0.5 \mathrm{~mm}$ de alto, las ascosporas son de color café, hialinas a amarillentas y algo más cortas y anchas i. e. de (34-)35.5-43(-44) x (6.5-)7-9 $\mu \mathrm{m}$ vs. (37-)38.5-48 x (4-)5-5.5 $\mu \mathrm{m}$. Lo anterior nos conduce a considerar al taxon antes descrito como una variedad de $A$. stellata que ha evolucionado para colonizar palmas.

Por otra parte, es interesante notar la presencia sobre los ascomas de la variedad propuesta de lo que parece un dotideal hiperparásito.

Astrosphaeriella tornata (Berk. et M. A. Curtis) D. Hawks. et Boise, Sydowia, Ann. Myc. 38: 119. 1985. Figs. 1F y $2 \mathrm{~A}$.

Ascomas individuales rara vez unidos por sus bases, cónicos, de 0.4-0.5 mm de alto $\times$ 0.6-0.8 mm de diámetro, errumpentes, con restos del substrato en la base a manera de bandas o lengüetas reflejas, dándole al conjunto la apariencia de una estrella. Textura carbonosa. Superficie lisa. Externamente de color café obscuro a negruzco con el área papilar más obscura, internamente negruzcos. Pseudotecio colapsado, ceroso, de 0.5-0.6 mm de diámetro. Ostiolos papilados. Ascos bitunicados, con la túnica interna constreñida en su parte superior, cilíndricos, octosporados en forma parcialmente biseriada, con pedicelos cortos, de 121-140 $\mu \mathrm{m}$ de longitud total x 11.5-13 $\mu \mathrm{m}$ de ancho, la parte esporígena de 112$131 \mu \mathrm{m}$, sin anillo apical o éste muy reducido y en tal caso inamiloide. Pseudoparáfisis trabeculadas, septadas, numerosas, de ca. $1 \mu \mathrm{m}$ de ancho, incluidas en una matriz gelatinosa. Ascosporas hialinas a amarillentas, de (37-)38.5-48 x (4-)5-5.5 $\mu \mathrm{m}$, fusiformes, equilaterales o inequilaterales con extremos aguzados, triseptadas, constreñidas a la altura de cada septo.

Espécimen estudiado: Chiapas, ejido Boca de Chajul, municipio de Ocosingo, sobre madera de una monocotiledónea en un acahual, 26.V.1988, San Martín 871 (ITCV): ibid., en madera de "caña brava" en la ribera del rio Lacantún, 27.V.1988, San Martín 781 (ITCV).

Observación: el material estudiado concuerda con $A$. tornata en el sentido de Hawksworth y Boise (1985). Dichos autores especularon sobre la posibilidad de que el 
San Martín y Lavín: Cuatro Especies y Una Variedad del Género Astrosphaeriella de México
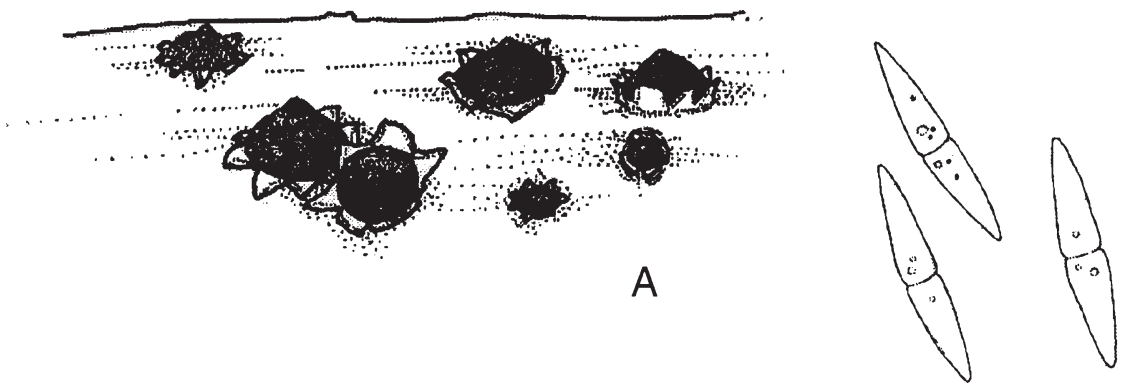

B
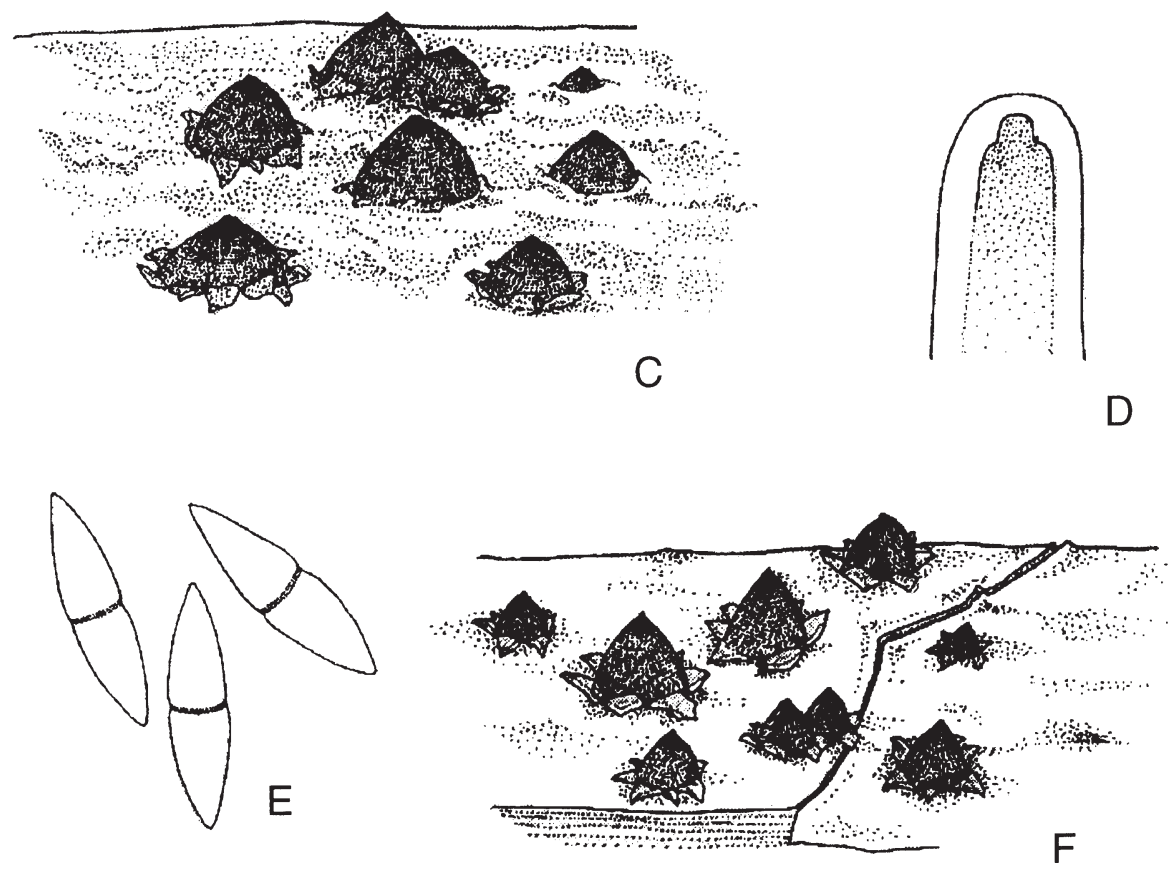

Fig. 1. A, B. Astrosphaeriella stellata: A. Ascomas errumpentes, $1 \mathrm{~cm}=800 \mu \mathrm{m}$; B. Ascosporas, $1 \mathrm{~mm}=1.8 \mu \mathrm{m}$. C, D, E. Astrosphaeriella stellata var. palmicola: C. Ascomas errumpentes, $1 \mathrm{~cm}=$ $1 \mathrm{~mm}$; D. Asco bitunicado, $1 \mathrm{~mm}=0.8 \mu \mathrm{m}$; E. Ascosporas, $1 \mathrm{~mm}=1.3 \mu \mathrm{m}$. F. Ascomas de Astrosphaeriella tornata, $1 \mathrm{~cm}=800 \mu \mathrm{m}$. 
hospedante de $A$. tornata fuera una palma. El material mexicano crece en madera de una gramínea bambusoide.

Astrosphaeriella tornata se conoce de Sri Lanka (Hawksworth y Boise, 1985) y de la Guayana Francesa (Cortecuisse et al., 1996).

Astrosphaeriella trochus (Penzig et Sacc.) D. Hawksw., Bot. J. Lin. Soc. 82: 46. 1981. Fig. 2B, C.

Ascomas individuales, cónicos, con el cuello alargado, de 0.5-0.9 mm de alto x 0.6$1 \mathrm{~mm}$ de diámetro, errumpentes, con restos del substrato en la base a manera de bandas delgadas, casi erectas, dándole al conjunto la apariencia de una estrella. Textura carbonosa. Superficie lisa. Externamente de color café obscuro, internamente negruzcos. Pseudotecio piriforme o colapsado, de 0.5-0.6 mm de diámetro. Ostiolos papilados, prominentes. Ascos bitunicados, con la túnica interna constreñida en la parte superior, cilíndricos, octosporados en forma biseriada, con pedicelos cortos, de 188.5-233.5 $\mu \mathrm{m}$ de longitud total $\mathrm{x}$ 13.5-17.5 $\mu \mathrm{m}$ de ancho, la parte esporígena de 169.5-208 $\mu \mathrm{m}$, sin anillo apical o éste muy reducido y en tal caso inamiloide. Pseudoparáfisis trabeculadas, septadas, numerosas, de ca. $1 \mu \mathrm{m}$ de ancho, incluidas en una matriz gelatinosa. Ascosporas de color oliváceo a café claro, translúcidas, de (59-)61.5-68 x (6-)6.5-7 $\mu \mathrm{m}$, fusiformes, inequilaterales con extremos aguzados, pentaseptadas, rodeadas de una envoltura hialina.

Espécimen estudiado: Chiapas, ejido El Rosario, municipio de Motozintla, sobre madera de Chusquea sp. en un bosque tropical con Pinus-Quercus, 19.X.1997, San Martín 6667Ch (ITCV).

Observaciones: el espécimen arriba descrito coincide con el concepto que para $A$. trochus tiene Hawksworth (1981). La especie ha sido citada de Africa, Asia, América del Sur; también se le ha detectado de Europa, donde aparentemente fue introducida de Asia.

Astrosphaeriella vaginata sp. nov. Fig. 2D, E, F.

Etimología: se refiere a la envoltura hialina que cubre a las ascosporas.

Ascomata singula, hemisphaerica vel conica, 0.4-0.5 mm alta $\times$ 0.4-0.6 mm diametro, erumpescentia cum hospitis vestigiis in superficiei. Textura carbonacea. Superficies laevis cum hospitis contexti vestigiis. Extus fusca vel nigricans, intus nigricans. Pseudothecia pyriformia, ceracea, 0.3-0.4 mm diametro. Ostiola papillata. Asci cylindrici, bitunicati, biseriatim octospori, cum tunica interna constricta in parte apicali, stipitibus brevibus, 137$163 \mu \mathrm{m}$ longitudine tota $\times 18.4-21 \mu \mathrm{m}$ lati, parte sporifera 125-141 $\mu \mathrm{m}$ longa, annulo apicali nullo vel reducto et inamiloideo in liquore Melzeri. Pseudoparaphyses trabeculosae, septatae, numerosae, circa $1 \mu \mathrm{m}$ latae, in matrice gelatinosa immersae. Ascosporae in statu maturo ex olivaceo hyalinae, (46.5-)49.5-56(-57) x (6.5-)7-9 $\mu \mathrm{m}$, fusiformes, equilaterales usque naviculares cum extremis acutatis, altitudine septi constrictae, tunica hyalina praeditae in statu maturo. 

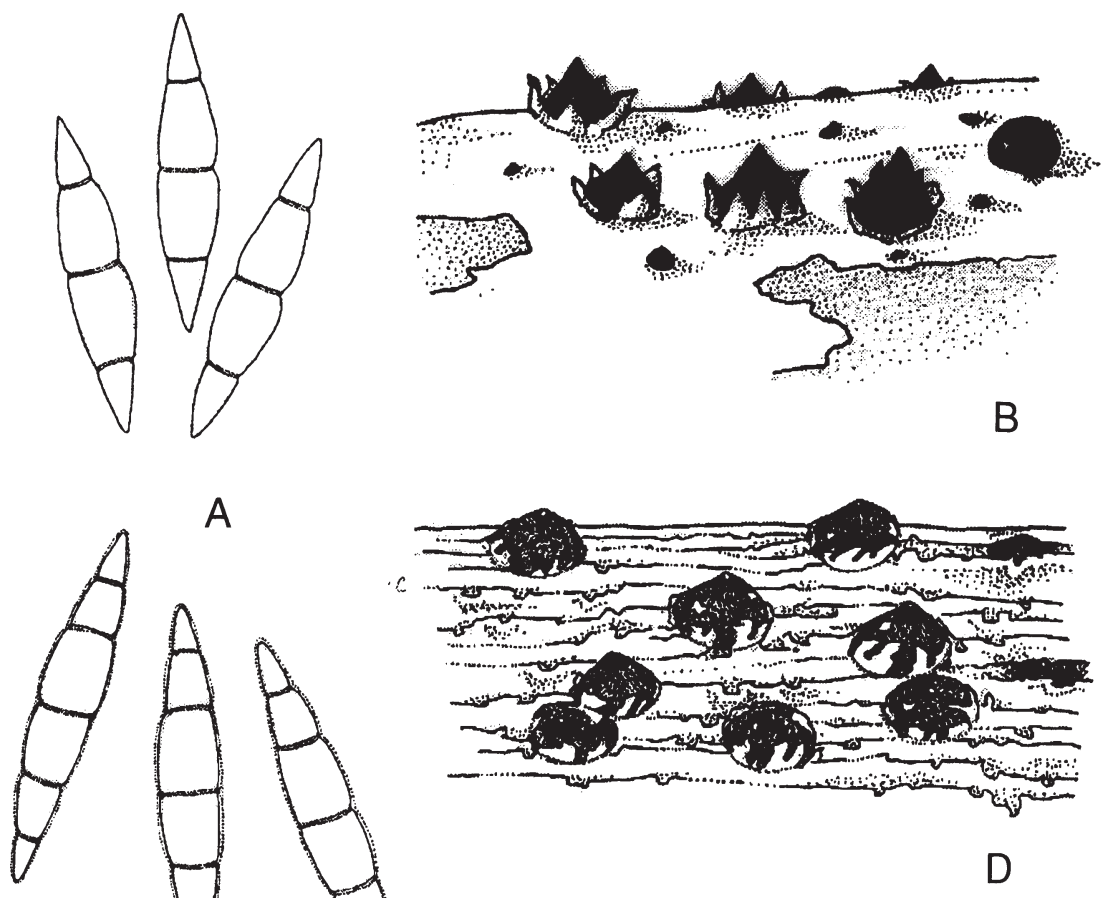

A
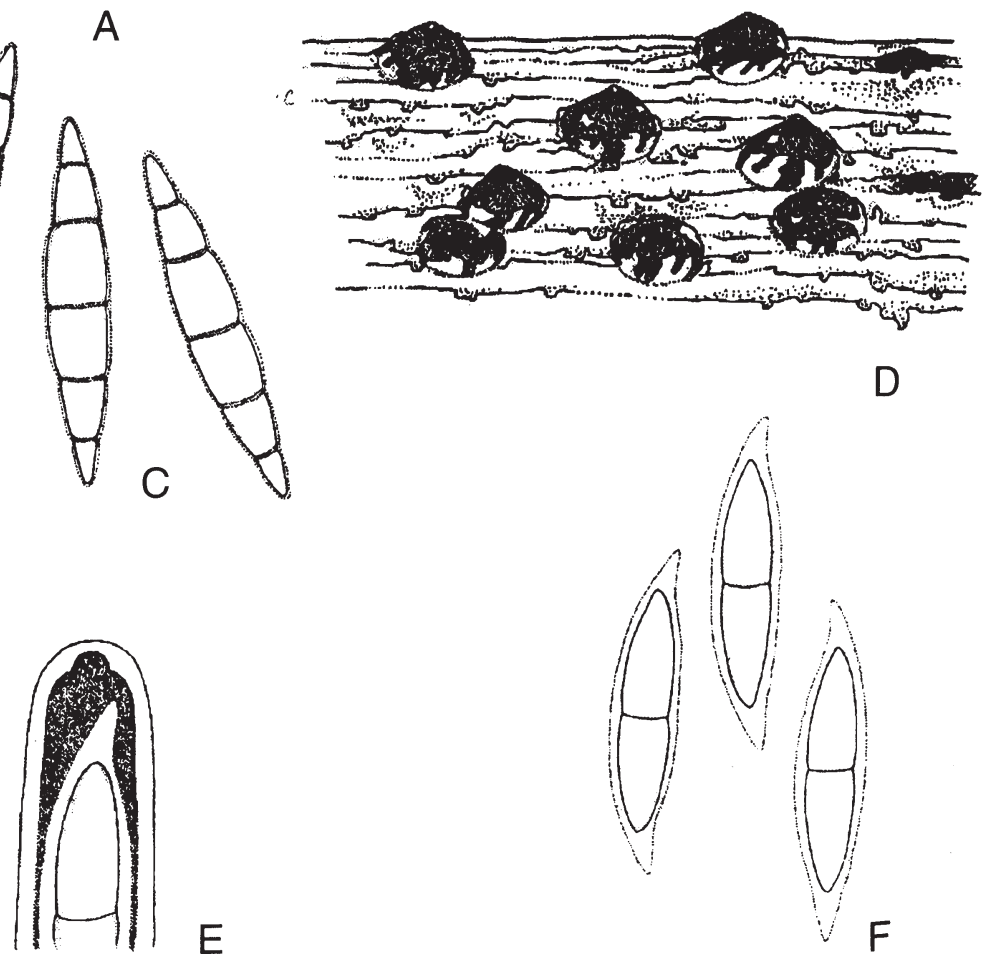

Fig. 2. A. Ascosporas de Astrosphaeriella tornata, $1 \mathrm{~mm}=1.3 \mu \mathrm{m}$. B, C. Astrosphaeriella trochus: B. Ascomas errumpentes, $1 \mathrm{~cm}=1 \mathrm{~mm}$; C. Ascosporas, $1 \mathrm{~mm}=1.6 \mu \mathrm{m}$. D, E, F. Astrosphaeriella vaginata: D. Ascomas con restos del hospedante adheridos a la superficie, $1 \mathrm{~cm}=400 \mu \mathrm{m}$; E. Asco bitunicado, $1 \mathrm{~mm}=1.5 \mu \mathrm{m}$; F. Ascosporas con una envoltura hialina, $1 \mathrm{~mm}=2 \mu \mathrm{m}$. 
Ascomas individuales, hemisféricos a cónicos, de 0.4-0.5 mm de alto x 0.4-0.6 mm de diámetro, errumpentes, con restos de substrato adheridos en la superficie. Textura carbonosa. Superficie lisa con restos de tejido hospedante. Externamente de color café obscuro a negruzco, con la región ostiolar más obscura, internamente negruzco. Pseudotecios piriformes, cerosos, de 0.3-0.4 mm de diámetro. Ostiolos papilados. Ascos bitunicados, con la túnica interna constreñida en la parte superior, cilíndricos, octosporados en forma biseriada, con pedicelos cortos, de 137-163 $\mu \mathrm{m}$ de largo total $\mathrm{x}$ 18.4-21 $\mu \mathrm{m}$ de ancho, la parte esporígena de 125-141 $\mu \mathrm{m}$, sin anillo apical o éste muy reducido y en tal caso inamiloide. Pseudoparáfisis trabeculadas, septadas, numerosas, de ca. $1 \mu \mathrm{m}$ de ancho, incluidas en una matriz gelatinosa. Ascosporas maduras hialinas con tintes oliváceos, de (46.5-)49.5-56(-57) x (6.5-)7-9 $\mu \mathrm{m}$, fusiformes, equilaterales a naviculares con extremos aguzados, constreñidas a la altura del septo, rodeadas de una envoltura hialina en la madurez.

Espécimen estudiado: Tabasco, alrededores del antiguo Colegio Superior de Agricultura Tropical, municipio de Cárdenas, en madera de Bactris baculifera Karw. ("jahuacté") en un acahual, 7.XI.1988, San Martín 1402 tb (HOLOTIPO: SMG).

Observaciones: esta especie es cercana a Astrosphaeriella aosimensis Hino et Katumoto, pero difiere en que sus ascosporas son mayores i. e. de (46.5-)49.5-56(-57) x (6.5-)7-9 $\mu \mathrm{m}$ vs. (28-)32-40(-42) x (4.5-)5-7(-8). Aunque ambos taxa fructifican a partir de madera de palmas, $A$. aosimensis lo hace en especies de Livistona, mientras que el taxon mexicano lo hace en una especie no identificada de Bactris. A. vaginata se parece a Javaria samuelsii Boise (Boise, 1984), pero difiere en que sus pedicelos ascales son más largos y al contacto con rojo congo los ascos no revelan anillo apical.

\section{AGRADECIMIENTOS}

Los autores agradecen especialmente al Dr. Rubén Durán, de Hemet, California, su participación en la revisión y corrección de la diagnosis en latin.

El primer autor patentiza su agradecimiento a los biólogos Arnulfo Moreno Valdez, Jorge Ayala Guajardo y Santiago Chacón Jiménez, por su gentileza y ayuda durante el trabajo de campo en Chiapas. Se agradece también al Sistema Nacional de Investigadores el apoyo para conducir estudios sobre la diversidad de los Ascomycetes de México.

\section{LITERATURA CITADA}

Aptroot, A. 1985. Redisposition of some species excluded from Didymosphaeria (Ascomycotina). Nova Hedwigia 60: 325-379.

Boise, J. 1984. New and interesting fungi (Loculoascomycetes) from the Amazon. Suppl. Acta Amazonica 14: 49-53.

Boise, J. 1985. An amended description of Trematosphaeria. Mycologia 77: 230-237. 
San Martín y Lavín: Cuatro Especies y Una Variedad del Género Astrosphaeriella de México

Courtecuisse, R., G. J. Samuels, M. Hoff, A. Y. Rossman, C. Cremers, S. M. Huhndorf y L. Stephenson. 1996. Check-list of fungi from French Guiana. Mycotaxon 57: 1-85.

Hawksworth, D. L. 1981. Astrosphaeriella Sydow, a misunderstood genus of melanommataceous Pyrenomycetes. Bot. J. Linn. Soc. 82: 35-59.

Hawksworth, D. L. y J. R. Boise. 1985. Some additional species of Astrosphaeriella, with a key to the members of the genus. Sydowia, Ann. Mycol. 38: 114-124.

Hawksworth, D. L., P. M. Kirk, B. C. Sutton y D. N. Pegler. 1995. Dictionary of the fungi. 8a. ed. Cambridge Univ. Press. Cambridge.

Holmgren, P. K., N. H. Holmgren y L. C. Barnett. 1990. Index Herbariorum. Part I: The herbaria of the World. The New York Botanical Garden. Nueva York. 693 pp.

San Martín, F. 1996. Una nueva variedad de Mycopepon smithii (Ascomycetes, Pleosporales). Acta Bot. Mex. 35: 9-12

Starback, K. 1895. Discomyceten-Studien. Kgl. Sv. Vetenskaps - Akad. Handl. Bihang 21: 1-42.

Aceptado para publicación en julio de 1998. 